\title{
Leitlinien der SGR zur Begutachtung rheumatologischer Krankheiten und Unfallfolgen
}

\section{Schweizerische Gesellschaft für Rheumatologie, Arbeits- gruppe Versicherungsmedizin, J. Jeger}

Leitlinien sind Handlungsempfehlungen. Sie dienen der Vereinheitlichung einer Dienstleistung und damit der Qualitätssicherung. Guidelines haben im Gesundheitswesen unter dem Einfluss der evidenzbasierten Medizin auf den verschiedensten Gebieten Einzug gehalten. Dafür sind viele Gründe verantwortlich, unter anderem die wachsenden Anforderungen an die Patientensicherheit und die knapper gewordenen Ressourcen der Gesellschaft.

In den Jahren 1990 bis 2002 zeigte sich ein dramatischer Anstieg der Anträge auf Versicherungsleistungen, insbesondere von Rentenanträgen bei der Invalidenversicherung. Seit 2003 ist die Zahl der Neurentner wieder rückläufig. Dabei sind psychische Störungen und Erkrankungen des Bewegungsapparates seit langem die Hauptgründe. Während im jüngeren Patientenkollektiv die psychischen Störungen überwiegen, so sind es bei den über 55jährigen Patienten die Erkrankungen des Bewegungsapparates, die am häufigsten zu einer vorzeitigen Berentung führen [1]. Die Störungen des Bewegungsapparates werden in der Regel von Rheumatologen beurteilt und begutachtet. Nach wie vor herrscht in der Schweiz ein Mangel an gut ausgebildeten Gutachtern.

Im Jahr 2004 hat die Schweizerische Gesellschaft für Versicherungspsychiatrie Leitlinien für die Begutachtung psychischer Störungen publiziert [2], die grosse Beachtung gefunden haben, und auf die das Bundesgericht in jüngeren Urteilen gelegentlich Bezug nimmt [3]. Die Arbeitsgruppe Versicherungsmedizin der Schweizerischen Gesellschaft für Rheumatologie hat nun Leitlinien für die Begutachtung rheumatologischer Krankheiten und Unfallfolgen verfasst, die wir hier vorlegen. Die Arbeitsgruppe setzt sich aus folgenden Mitgliedern zusammen:

- Prof. Dr. med. Peter Villiger, Direktor der Klinik für Rheumatologie, Inselspital Bern, Präsident der Schweizerischen Gesellschaft für Rheumatologie;

- Dr. med. Pius Brühlmann, Leitender Arzt, Rheumaklinik Universitätsspital Zürich;
- Dr. med. Dieter Frey, praktizierender Rheumatologe, Basel;

- Dr. med. Thomas Meyer, Leitender Arzt Rheumatologie, Kantonsspital Baden;

- Dr. med. Hans Schwarz, Chefarzt Rheumatologie, Bethesda Spital Basel;

- Dr. med. Jörg Jeger, Chefarzt MEDAS Zentralschweiz (Leiter der Arbeitsgruppe).

Die Leitlinien wurden vom Vorstand der Schweizerischen Gesellschaft für Rheumatologie in der Sitzung vom 27. September 2006 genehmigt. Sie sollen eine Hilfestellung sein für alle Rheumatologinnen und Rheumatologen, die gutachterlich tätig sind, aber auch für die Ärztinnen und Ärzte in der fachärztlich rheumatologischen Ausbildung.

Die Tätigkeit im Grenzgebiet zwischen Medizin und Rechtsanwendung erfordert in vieler Hinsicht ein eigenständiges Vokabular, das für den Anfänger oft schwierig zu verstehen ist und häufig zu Missverständnissen führt. Wir haben aus diesem Grund ein ausführliches Glossar integriert, das die gängigsten Begriffe erklärt, die der Gutachter als Rüstzeug für seine Tätigkeit benötigt. Jurist und Mediziner müssen sich verstehen können, obwohl sie eine Sachlage aus verschiedener Denk- und Sichtweise - deduktiv bzw. induktiv - zu beurteilen gewohnt sind. Herrn Prof. Dr. iur. U. Meyer, Richter an der Sozialrechtlichen Abteilung des Bundesgerichtes in Luzern, danken wir herzlich für die kritische Korrektur der Leitlinien aus juristischer Sicht.

\section{Literatur}

1 Bundesamt für Sozialversicherung. IV-Statistik 2006. www.bsv.admin.ch.

2 Leitlinien der Schweizerischen Gesellschaft für Versicherungspsychiatrie für die Begutachtung psychischer Störungen. Schweiz Ärztezeitung 2004;85(20):1048-51.

3 Urteil des Eidgenössischen Versicherungsgerichtes U 391/06 vom 9.8.2006. www.bger.ch. 